\title{
ROLE AND IMPORTANCE OF COMMUNICATION IN TRANSDISCIPLINARY RESEARCH MANAGEMENT
}

\author{
Oksana Kushnir \\ Department of Philosophy \\ Yuriy Fedkovych Chernivtsi National University \\ 2 Kotsyubynsky str., Chernivtsi, Ukraine, 58012 \\ oksana.kushnir.93@ukr.net
}

\begin{abstract}
This paper is devoted to studies of communication process peculiarities, role of "negotiators" in transdisciplinary studies and also interaction processes between science and practice on an example of an empirical case. Based on the conducted analysis, a hypothesis has been confirmed that effective realization of the transdisciplinary approach is grounded on effective communication, and factors, worth attention for successful realization of research aims in transdisciplinary projects have been determined: balance of power relations between participants of transdisciplinary studies, choice of mediators' groups, change of initial instructions of scientists, cultural differences and their influence on general organization of processes, choice of communication types and forms. A necessity to construct a communicative strategy of a transdisciplinary project, forming a common field for effective interaction, has been determined; at that there must be taken into account group development stages, key topics of communication and its success indicators. It has been proved, that communication management is a necessary element of transdisciplinary studies organization and conduction. For improving communication quantity and quality, it is necessary for scientists and stakeholders to take equal responsibility and to participate in decision making.
\end{abstract}

Keywords: communication, transdisciplinarity, transdisciplinary project, stakeholders, negotiator, mediator.

DOI: $10.21303 / 2504-5571.2021 .001626$

\section{Introduction}

The modern stage of science development is remarkable for a structural change of knowledge production, correlating with tendencies of sociocultural processes, often described as the "world without boundaries". First of all, it is about globalization processes, which projections are manifested in socioeconomic convergence of the world order, widening of information-communication networks, formation of transnational economic organizations and management structures, aimed at achieving a certain global consensus, senses of stable being. Today science pays more and more attention to overcomplicated, human-measured, live and social systems. As far as the fundamental world level goes beyond the horizon of possibilities of hypotheses experimental verification, science must a bit change a sphere of cognitive activity, to replace the focus of its interests on information and high technologies, medicine, gene engineering, economics, branches with non-distinct prognoses and high risks, to become transdisciplinary.

As opposite to disciplinary and interdisciplinary methods, serving as cognition instruments in a concrete science, formed based on ontological and gnoseological ideas about research objects, a base of transdisciplinary methods is theories, generating from the integral "world picture". Such "transdisciplinary" world picture allows to elaborate new research methods of an object and also to interpret knowledge, obtained by disciplinary and interdisciplinary ones. But it must be noted, that ideas about transdisciplinarity are first of all connected not only with "universal" science methods, but with a phenomenon of science interaction with other forms of the human living world, when a transfer beyond science itself takes place, and its projections in everyday life and other forms of culture are studied. Under such conditions public takes more and more active participation in discussion of urgent ecological and political problems, initiating and practically realizing transdisciplinarity mechanisms. It is interpreted not only as a transfer beyond separate scientific disciplines, but beyond separate cultures, nationalities, states. As B. Nicolescu notes it, "transcultural and transnational approaches to reality" are used more and more often [1].

The German sociologist of science P. Vainhart notes, that an essential indicator of changes in modern science is the formation of co-called hybrid communities [2]. It is about organizational structures, "where scientists, politicians, administrators, representatives of industry and other in- 
terest groups directly cooperate for determining a problem, research strategy and finding its solution. It includes the process of transferring political aims into technical ones and research strategies that bind different discourse universes" [2].

Summarizing the aforesaid, let's outline one of most important aspects of transdisciplinary studies - orientation on production and dissemination of knowledge in the process of active communication. Transdisciplinary communication is realized by both formal and informal channels, and the organizational structure of the new type of networks, appearing at that, determines the specificity of knowledge itself. The formed transdisciplinary communication network keeps its topicality also after solving a problem and partial change of the context of its actualization and understanding. Discussion of a problem in wide public circles, involving socially active actors, studying important factors of its appearance and overcoming ways result in further dissemination of obtained knowledge not only by such traditional channels of scientific communication as conferences and scientific journals, but first of all at practical activity and communication of specialists in correspondent branches and social spheres. As F. Harris and F. Lyon state it, "for effective communication and successful research conduction, a transdisciplinary team must be rather big for covering different types of knowledge, but rather small for its members can build relations with each other" [3].

Special attention is paid to the problem of communication in transdisciplinary projects in works by F. Balsiger, E.T. Hall, M. O'Rourke and K. Liu. The final aim of communication in transdisciplinary studies, according to F. Balsiger, "is in achieving joint knowledge generation by a group of scientists and stakeholders" [4], and E.T. Hall and M. O'Rourke note that "transdisciplinary communication is best fixed in the sociocultural conception of communication as joint generation of a sense for attaining an aim" [5]. K. Liu accents attention on the cultural aspect and its results that, from his point of view, must be taken into account in organization and conduction of intercultural transdisciplinary studies. The scientist notes that "difficulties and even conflicts in communication appear in different groups of people from different cultural contexts, especially from the east and west, because they don't understand each other in their intercultural communication" [6]. Taking into account the aforesaid, one can make a conclusion that the problem of communication in transdisciplinary studies gains essential scientific-methodological, philosophical importance and topicality. Further analysis of stable features of transdisciplinarity, and management of transdisciplinary projects needs substantial studies of the communication process, mediators' role in transdisciplinary studies and also interaction processes between theory and practice. But, as the scientific search demonstrates it, there are essential gaps in realization of these tasks.

Let's note main obstacles on this way:

1) there is no united communicative platform and conventional base for integration and cooperation between different disciplines, knowledge types and scientific approaches till today;

2) successfulness of communication problem solving in transdisciplinary studies in practice remains a bit behind theory because of sophisticated nuances, influencing the communication, mutual understanding and cooperation effectiveness.

These factors demonstrate the necessity to build a certain communicative framework and to elaborate practical recommendations as to transdisciplinary project management by improving the communication process, because communication characteristics of transdisciplinarity are fully manifested just in the process of concrete transdisciplinary projects and studies.

The aim of the work is to study communication process peculiarities and also interaction processes between science and practice on an example of an empirical case.

\section{Materials and Methods}

The analysis of transdisciplinary processes on an example of the empirical case as to complex land use in southeastern China, conducted by the international transdisciplinary team of German and Chinese scientist and also local stakeholders, has been realized. A source of information about this study became publication [7]. The project "Stable caoutchouc cultivation in the Mekong region: elaboration of the conception of complex land use in the Chinese province Yunnan" was financed by the Federal ministry of education and researches of Germany within the program of 
complex land use. The German-Chinese working team consisted of nine subdivisions, oriented on studying ecological (soils, water biodiversity) and socioeconomic (estimation of living conditions, farmers' life activity) problems.

Alongside with scientists, involved in the interdisciplinary cooperation, wide circles of stakeholders, especially representatives of Chinese state bureaus and farmers, actively participated in the project. For setting up communication and interaction of all parties, a special group of specialists-mediators was involved into the research. They played the role of a kind of "bridge", "negotiator" between German researchers and stakeholders from China, favoring the effectiveness improvement of their cooperation. The activity of this group became a base of a separate subproject - "knowledge translation management" [7]. As far as for conducting the research, intensive communication between different research subgroups was necessary for integrating knowledge of scientists and local stakeholders, we can trace on this example peculiarities of such projects realization, taking communication problems and ways of their solving in the process of transdisciplinary team formation and development, based on the five-step model of working groups development, offered by B. Tuckman [8], as a base on the analysis.

\section{Results}

The first stage - team formation - provided the meeting of participants and conceptualization of the main research task. Its main problem was in the fact that from the very beginning Chinese stakeholders manifested certain distrust to potential partners, hesitated about participation in the project, so manifested no activity. For overcoming this problem, the group, appointed for facilitating communication processes, has formed two strategies: at first, they got access to potential project participants through personal profiles in social networks and met with them unofficially for demonstrating the research expedience and for motivating interest. At second, they have raised the status and authority of the seminar by sending stakeholders official letters, signed by the wellknown local partner [7]. These strategies allowed to interest and to unite all project parties and to determine the common orientation on further cooperation.

At the next stage - «storm» - the interaction between the participants intensified, conflicts appeared, institutionalization of power relations between both separate participants and subgroups took place, based on their solving. Alongside with fundamental discordances between representatives of different disciplines on ontological, gnoseological and methodological questions, intercultural communication between German researchers and Chinese stakeholders remained one of main problems. For motivating local stakeholders to the active participation, the mediators' team stimulated scientists to share more useful information with them, using such additional communication channels as leaflets, information brochures and presentation of previous research results at seminars. The use of Chinese language alongside with German and English on the project website, protocols and so on also has a positive influence [7]. These arrangements turned out effective for improving communication between scientists and stakeholders, but needed much more time and human resources than planned.

Most scientists found it an optimal variant to conduct their disciplinary scientific studies without interactive arrangements, such as seminars with stakeholders. Some of them hesitated, is it at all necessary to cooperate with groups of parties actively, as it is needed by the context of a transdisciplinary research. At initial research stages scientists had no unity as to importance of interaction with actors from the nonacademic environment. Scientists have changed their attitude to the problem and joined interactive discussions, seminars and focus-groups because of continuous efforts of the working group on communication management and pressure from external estimators of the project, insisted on the necessity of cooperation between scientists and practicians.

The third stage - stabilization or regulation of group relations. At this stage there takes place team development, intensification of solidarity and open exchange of information, resources and so on. Conflicts that had appeared at the storm stage were gradually solved by intensified communication. The most important problem, appeared at this stage, is different understanding of the system of land exploitation. Team members looked at this situation from different perspectives, conditioned by the dynamic social context, complicated power relations and interests of different 
participants of the project [7]. The analysis of this separate case, described by results of the transdisciplinary research, conducted in China, proves well-known facts as to the influence of cultural directives on scientific research practice. Regular informal discussions, meetings between scientists and stakeholders turned out a strong instrument for setting up communication and interaction, allowing all parties fully speak about their minds and favoring the exchange of necessary information, in such a way facilitating mutual understanding and activating learning.

The final stage is research realization, within which, according to certain scientists, the main attention must be accented on integration of knowledge of transdisciplinary team members. The method of scenario building is called an effective instrument for that [9]. But obvious differences in scientists' and practicians' understanding of the offered method of knowledge integration have been revealed. For overcoming the gap in the group interaction, mediators at first had discussed with stakeholders their expectations from the research and quantitatively represented the obtained information in scientific modeling, then transferred the scientific scenario in qualitative plot lines for its simplified understanding by stakeholders. It was necessary to repeat this procedure several times for setting up communication. When parties reached certain understanding, they exchanged ideas. After discussion, the team has determined a priority scenario that demonstrated expected results for local policy and arrangements for land use improvement [7]. Especially, one of elements of the commonly elaborated scenario was a consensus as to giving priority ecosystem services: control on water quality and soils erosion. Correspondingly, concrete solutions were discussed (for example, a plan of water protection) [7]. After finishing the work on the project, it was expected that commonly determined priorities of further land use would be used in practice.

So, the analysis of group processes gives detail information about how team members interact and react to tasks, and also how negotiators' (mediators', communicators') activity favors effective solving of problems, caused by weak or insufficient communication in transdisciplinary studies. Although conflicts are inevitable, observations on relations development and tasks realization help to trace the source of the problem: problem relations between people or a task itself. The analysis of communicative aspects of the empirical case - project "Stable caoutchouc cultivation in the Mekong region: elaboration of the conception of complex land use in the Chinese province Yunnan" - confirms a hypothesis that effective communication is a base of effective realization of the transdisciplinary approach.

Based on the conducted analysis, factors that must be taken into account for successful realization of research aims in transdisciplinary projects have been determined.

At first, power relations between subjects of a transdisciplinary team must not be ignored. In transdisciplinary projects scientists often consider themselves a group with more power that guides the research process. Such power asymmetry may threaten interaction between science and practice. Participants with more power are inclined to treat participants with less power authorities with less respect, attention and openness. The last ones, in their turn, strive to give their colleagues changed or incomplete information. Such negative communication often intensifies the visible power hierarchy and asymmetry. For solving this question, it is necessary to understand that both scientists and stakeholders are equally important participants of a project, and not to treat scientists as a dominant group that involves local stakeholders, giving them a service. But it is difficult in practice, because transdisciplinary projects are often begun and planned by groups of scientists, and a voice of parties' representatives is absent at the stage of presenting an application for a project. At the same time just the opposite situation, for example, when companies, state laboratories, local authorities, industrial sector search for new ways of cooperation with academic research groups may be observed. In this case just they feel own advantage. That is why more attention must be paid to balancing power relations between participants of transdisciplinary studies for creating a base for mutually profitable interaction and information exchange. For stimulating equality at communication at the stage of group formation, it is also important to determine communication rules, to fix rights and duties of team members formally in written form (for example, to conclude agreements and to prepare protocols).

At second, more attention must be paid to mediators' group, allocated for facilitating communication processes. In transdisciplinary studies they play a role of some "bridges", uniting dif- 
ferent cultures, languages, knowledge types and professions. Their main task is, from one side, to transfer research results in an understood, verisimilar form, potentially useful for stakeholders, and from the other one, - to inform scientists about interests and problems of other group participants. There appears a question: who can play the role of negotiator in such studies? Ones propose external facilitators or trained mediators, others suggest that sociologists can play this role within a project. But sociologists can provide only elucidation of problems of human behavior and effective communication that may be a cause of role conflicts or instructions. External professionals can provide a "neutral" territory for cooperation, but their efforts may be insufficient, because they are not familiar with a work specificity of both scientists and stakeholders and also with a content of a transdisciplinary problem at disciplinary and practical levels. Project managers must chose negotiators according to concrete conditions of each study. Negotiator is not obligatorily the same person or group during the whole process, most likely, a series of communicative roles may be realized by different persons at different stages. Actors, most fit for concrete circumstances, may perform these roles. At the same time each participant of a team takes responsibility for a contribution, made in negotiations, discussions and facilitation of the common work.

At third, the importance of changes of scientists' initial directives must be underlined. They often understand transdisciplinary communication as an additional, not necessary means. It is turned out, that having mastered transdisciplinary research, a scientist may spend many years for developing respect to other knowledge branches and professions and communicative skills. It is much easier for researchers to change their views, if a paradigm of their professional training was not only professionally oriented, and they mastered so-called soft skills just during university studies. At the beginning of the project that became an object of the analysis most researches didn't understand the importance of intensive and regular communication with stakeholders. Being involved in researches, they were not ready to "waste time" for communication and setting up of cooperation. External parties, interested in project realization, especially, estimators of presented applications, on the contrary, insisted on the necessity to make efforts for organizing internal group communication that the effectiveness of cooperation and final result depend on. For eliminating obstacles in communication, the group of mediators has conducted a lot of events, motivating the active exchange of ideas, knowledge and experience between scientists and stakeholders. As far as communication is set up gradually, it needs continuous efforts during the whole project term. Revolutionary changes are possible, but they take place only as a result of stable interventions. The most important task is to form and to support participants' and subgroups' mutual confidence, to motivate openness in communication, taking into account the cultural context. For keeping the desirable working atmosphere in a group, it is necessary to involve specialists, closely acquainted with communicative-managerial processes, to continuous work, but it is often not taken into account at organizing transdisciplinary researches.

At fourth, cultural differences and their influence on general organization of processes, realization of separate activities, creation of correspondent conditions for communication and cooperation in international studies that are intercultural, transdisciplinary, must be taken into account.

In fifth, "negotiators" must pay special attention to the choice of communication forms and types. As it has been already noted earlier, the choice of communication means is conditioned by both the specificity of a concrete task and cultural, professional and other context. The project analysis has confirmed the hypothesis about intensification of the role of informal interprofessional communication. Less formal communication ways facilitate information exchange, simplify mutual understanding, favor new ideas and the main aim realization - generation of common transdisciplinary knowledge. New forms of scientific communication, combining direct personal communication and one, mediated by modern means, also appear in such projects. E-means of communication allow scientists to find experts from any point of the planet, to consult with them. Due to the internet, new publications circulate fast, scientific information becomes accessible not only for specialists. Scientists find communication channels with authorities, business, public easier, get fast feedback as to scientific discoveries. It became possible to conduct scientific studies, needing essential human resources. Rather fast scientific communication technologies serve not a 
means, but a base of communication: internet-platforms, uniting all persons, groups, organizations, potentially interested in cooperation, appear.

Having analyzed the described empirical case, one can make a conclusion that communication is a base of the transdisciplinary approach. Its practical realization is impossible without taking into account communicative factors in the transdisciplinary methodology.

\section{Discussion}

In fact, a lot of examples of transdisciplinary studies in international practice have been described. Thus, the project "Strengthening of food and nutrition safety by integration of poultry and crops in Tanzania and Zambia" united specialists in animal husbandry, agriculture and health protection, economists, ecologists, sociologists and practicians [10]. This five-year project was financed by the Australian center of international agricultural studies and realized by the University of Sidney (faculty of veterinary sciences, Faculty of agriculture and external environment and the School of public health) in cooperation with groups of parties from Tanzania and Zambia [10].

Quality improvement of food and nutrition remains one of global problems, which solving needs the complex approach for achieving long-term aims and sustainable development. The absence of progress in the desirable direction in the last decades was observed by the international community in the African countries to the south from Sahara, where the population increment resulted in the increase of the total number of people, suffering from malnutrition [11]. Despite regular agricultural studies, purposeful health protection and introduction of the nutrition program, the prevalence of the problem of development delay in children younger 5 years in Tanzania and Zambia is estimated as $42 \%$ and $40 \%$ respectively [12], that is directly or indirectly connected with malnutrition.

Such problems as products' quality, nutrition and malnutrition in childhood are called "malicious problems", they go beyond complication limits, and their studying and solving need transdisciplinary approaches. V.A. Brown defines "malicious problem" as "such, not subjected to a final solution, because any solution generates further problems, and it is neither true nor false, neither good nor bad, but the best that can be found under given conditions in the concrete time moment" [13].

This project needed understanding of the complex character of the set problems and the ability to work effectively in complex schemes. In this context knowledge and experience exchange between scientists and stakeholders was a difficult task itself. For simplifying the situation, a communicative strategy, within which communication acted both as a direct dialogue and a process, leading to unpredictable and unexpected results, needing from team members a high flexibility and adaptability level, has been elaborated.

Mains tasks of communication within this project were in supporting the research aims and connected results and also in favoring interaction between all research participants. Communication allows to determine attitudes, understanding and needs of each involved player and, based on it, to form explications, recommendations and activity, best corresponding to common interests.

A communication strategy in this context is a dynamic structure, needing association and cooperation at realizing the project. Communication processes were destined for:

- providing distinct understanding of aims, tasks and results of the project by the parties' groups and also scientific community and correspondent state institutions;

- favoring information exchange and creation of collective knowledge;

- favoring effective interaction within the transdisciplinary team;

- creating a safe space for ideas exchange, discussion and solving of questions;

- supporting mechanisms of integrative, reflexive and estimation approaches, allowing to realize continuous education and adaptation and also to reveal and to learn new ideas and strategies;

- informing the correspondent parties about successes and results, obtained during the project [10].

A series of recommendations as to increasing the communication process has been elaborated. Among them:

- formation of collective senses, taking into account knowledge, ideas and propositions of all research participants; 
- creation of a base for collective production of knowledge and their exchange;

- use of different cognitive approaches and methods for creating a united whole;

- involvement of participants to elaboration and realization of the project;

- adaptation of the complex system approach;

- elaboration of a scenario of relations with stakeholders;

- openness to different approaches, methodologies and new practices [10].

It is extremely important to create a certain platform, base for interaction between all team participants that will be effective during the whole project life. At any stage of the research process it may become urgent to study new strategies, because new unpredictable elements, opening both certain perspectives and threats for team development, appear. They need continuous analysis and also long-term planning of communication, using which it is possible to correct an initial strategy, rejecting unrealized ideas.

The analysis of the empirical case has demonstrated that effective realization of the transdisciplinary approach is grounded on effective communication, factors, worth attention for successful realization of the research aims in transdicsiplinary projects have been determined and a series of recommendations as to increasing the communication process efficiency in transdisciplinary projects has been presented. But the aforesaid factors and recommendations may change according to research aims and working team participants, because each transdisciplinary project is unique and each time provides interaction of representatives from both different scientific branches and extrascientific knowledge. Further studies may be directed on revelation of improvement factors of support systems and institutional mechanisms of transdisciplinary communication.

\section{Conclusions}

So, the transdisciplinary approach may be used for solving different problems, such as land use and food products quality improvement or others that have the one common characteristic complexity and difficultness. And despite the specificity of studying these problems, it remains obligatory to build a communication strategy of a transdisciplinary project that forms a common field for effective interaction. At building this strategy, there must be taken into account group development stages, key communication topics and its success indicators. At each stage of the working team development general questions that must be considered in each subgroup, concrete questions, considered by the whole transdisciplinary team, appear, and continuous monitoring of cultural aspects of intercultural interdisciplinary cooperation is conducted.

Continuing the idea of B. Tuckman, we recommend to take into account indicators of delimitation of social and research tasks at group development stages. They may be used for determining communication successfulness in a transdisciplinary team, in other words, is a set aim achieved at each stage in the aspect of relation development and decision making. Such indicators can help managers, facilitators or mediators of transdisciplinary projects to understand the dynamics of team formation and, having analyzed the process of tasks realization, to diagnose conflicts and problems distinctly and to use correspondent preventing measures or instruments of their overcoming at each stage.

It must be noted, that groups often don't pass the expected succession of stages in practice and repeatedly undergo one or several of them. Under such conditions transdisciplinary communication also doesn't develop in a certain direction, but contains closed cycles that may have both positive and negative influence on the whole process. The positive effect is observed only if parts or stages repeat for widening and deepening knowledge. For estimating and foreseeing results of such iteration, it is necessary to reveal cyclic actions and to take into account feedback and, if necessary, to correct the process correspondingly. Due to active and continuous communication and interaction during the whole process duration, all or most participants can reach results, satisfying their demands and corresponding their expectations. It is possible to build such cooperation on strong and long-term relations, openness and trust of team members. Before planning a team work, it is necessary to take into account strong and weak sides of an actual communicative infrastructure and also to get acquainted with participants for choosing a maximally effective form of communication with them. 
Summarizing, it is worth noting, that solving difficult, complex problems needs intensive and effective communication with different groups of parties for integrating scientific and practical knowledge in transdisciplinary projects. The base of effective cooperation is mutual understanding and empathy, needing scientists' and stakeholders' readiness to communication, cooperation within different disciplines, cultures, time costs, necessary for them. More attention must be paid to team building in transdisciplinary projects, attracting contacts just before the beginning of work with a project. Communication management is a necessary element of transdisciplinary studies organization and conduction. For improving communication quality and quantity, it is necessary for scientists and stakeholders to take equal responsibility and to participate in decision making. The analysis of group processes and their dynamics will help transdisciplinary team subjects to understand mutually connected cooperation links and correspondingly to facilitate and to raise the effectiveness of group work at realizing different tasks.

\section{References}

[1] Nicolescu, B. (1997). The Transdisciplinary Evolution of Learning. In Proceedings of the International Congress on What University for Tomorrow? Towards a Transdisciplinary Evolution of the University. Locarno, 1-11. Available at: https://www. scirp.org/(S(351jmbntvnsjtlaadkposzje))/reference/ReferencesPapers.aspx?ReferenceID=1592993

[2] Vainhart, P.; Arzakanian, Ts. H. (Ed.) (1989). The relationship between science and technology: a sociological explanation. Fylosofyia tekhnyky v FRH. Moscow: Prohress, 131-161.

[3] Harris, F., Lyon, F. (2013). Transdisciplinary environmental research: Building trust across professional cultures. Environmental Science \& Policy, 31, 109-119. doi: http://doi.org/10.1016/j.envsci.2013.02.006

[4] Balsiger, P. W. (2004). Supradisciplinary research practices: history, objectives and rationale. Futures, 36 (4), 407-421. doi: http://doi.org/10.1016/j.futures.2003.10.002

[5] Hall, T. E., O'Rourke, M. (2014). Responding to communication challenges in transdisciplinary sustainability science. Transdisciplinary Sustainability Studies: A Heuristic Approach. Routledge: Oxford, 119-139.

[6] Qingxue, L. (2003). Understanding Different Cultural Patterns or Orientations Between East and West. Investigationes Linguisticae, 9, 21-30. doi: http://doi.org/10.14746/i1.2003.9.3

[7] Blagodatskiy, S., Langenberger, G., Cadisch, G. (2018). An Integrated Assessment Framework for Natural Rubber Plantations. München: Grin Verlag, 172.

[8] Tuckman, B. W. (1965). Developmental sequence in small groups. Psychological Bulletin, 63 (6), 384-399. doi: http://doi. org $/ 10.1037 / \mathrm{h} 0022100$

[9] Kok, K., Bärlund, I., Flörke, M., Holman, I., Gramberger, M., Sendzimir, J. et. al. (2014). European participatory scenario development: strengthening the link between stories and models. Climatic Change, 128 (3-4), 187-200. doi: http://doi. org/10.1007/s10584-014-1143-y

[10] Strengthening food and nutrition security through family poultry and crop integration in Tanzania and Zambia (2014). The Australian International Food Security Centre (AIFSRC). Available at: https:/aifsc.aciar.gov.au/projects/strengthening-food-and-nutrition-security-through-family-poultry-and-crop-integration.html

[11] The State of Food Insecurity in the World (2015). Available at: http://www.fao.org/3/a-i4646e.pdf

[12] Tanzania Demographic and Health Survey (2010). Available at: https://dhsprogram.com/pubs/pdf/FR243/FR243[24June2011].pdf

[13] Harris, J. A., Brown, V. A., Russell, J. Y. (Eds.) (2010). Tackling wicked problems through the transdisciplinary imagination. London: Earthscan, 332. 